\title{
La participación estudiantil en el Instituto Autónomo de Ciencias y Artes del estado de Oaxaca y la huelga de 1936
}

Student Participation in the Autonomous Institute of Sciences and Arts of the State of Oaxaca and the 1936 strike

\author{
Alejandro Arturo Jiménez Martínez \\ Instituto de Ciencias de la Educación de la Universidad \\ Autónoma "Benito Juárez" de Oaxaca \\ aajm25@hotmail.com
}

\begin{abstract}
Resumen
En 1936, el Instituto Autónomo de Ciencias y Artes del Estado de Oaxaca (IACAEO) vivió una huelga que transformó sus relaciones con los poderes públicos local y federal. En este artículo se muestra la formación participativa y organizativa de los estudiantes del Instituto y sus repercusiones en el movimiento estudiantil. La huelga sucedió en el contexto de la disputa generada después del Primer Congreso de Universitarios Mexicanos de 1933 y el intento de implementar el socialismo en las instituciones de educación superior del país. La Federación de Estudiantes Oaxaqueños (FEO) sostenía las orientaciones de la Confederación Nacional de Estudiantes (CNE), cuyo programa pugnaba por la autonomía de las instituciones de educación superior del país. Sin embargo, el Comité de Huelga fue sensible al socialismo de organizaciones estudiantiles e instituciones, y en sus documentos combinó la lucha por la autonomía y el discurso socialista de la época.
\end{abstract}

Palabras clave: educación socialista, autonomía, huelga estudiantil, Instituto Autónomo de Ciencias y Artes del Estado de Oaxaca.

\section{Abstract}

In 1936 the Instituto Autónomo de Ciencias y Artes del Estado de Oaxaca (IACAEO) experienced a strike that transformed its relations with the local and federal public powers. The article shows the participatory and organizational training of the students of the Institute and the repercussions of that in the student movement. The strike occurred in the context of the dispute generated after the First 
Congress of Mexican University Students of 1933 and the attempt to implement socialism in higher education institutions in the country. The Federación Oaxaqueña de Estudiantes (FEO) supported the guidelines of the Confederación Nacional de Estudiantes (CNE), whose program struggled for the autonomy of Mexican higher education institutions. However, the strike committee was sensitive to the socialism of student organizations and institutions and in its documents combined the struggle for autonomy and the socialist discourse of the time.

Keywords: Socialist education, Autonomy, Students' Strike, Instituto Autónomo de Ciencias y Artes del Estado de Oaxaca.

\section{Introducción}

El Instituto Autónomo de Ciencias y Artes del Estado de Oaxaca (IACAEO) abrió sus puertas el 8 de enero de 1827 y durante el siglo XIX formó a liberales oaxaqueños que contribuyeron en la construcción del estado nacional mexicano, entre los que destacan Benito Juárez, Porfirio Díaz, Ignacio Mariscal, Matías Romero y Emilio Rabasa, entre otros. En aquella época, dicha casa de estudios se desarrolló en un ambiente en el que primaban las costumbres católicas, por lo que, aun cuando proveyó al gobierno federal y estatal de brillantes hombres de estado, se afirma que su ideología "no logró penetrar en la sociedad" oaxaqueña (Lempérière, 1994: 91).

El proceso revolucionario que se vivió en el país entre 1910 y 1920 provocó en Oaxaca inestabilidad en el poder ejecutivo local, la instauración de un gobierno que se declaró soberano respecto del régimen constitucionalista de Venustiano Carranza y, al final, una negociación entre la élite oaxaqueña y el grupo sonorense que en 1920 obtuvo el poder nacional. Este proceso generó en el IACAEO un estado de vulnerabilidad. En 1916, el gobierno carrancista que ocupó la ciudad de Oaxaca intentó cerrar la casa de estudios, pero profesores y alumnos consiguieron mantenerla abierta sin el apoyo estatal.

En 1921, el gobierno revolucionario de Manuel García Vigil retomó su responsabilidad con el Instituto. A partir de entonces, inició un periodo en el que es posible percibir una comunidad con importantes carencias económicas, pero con una vida interna rica en experiencias dentro y fuera de sus aulas que permitieron la formación intelectual y política de su comunidad.

En la ciudad de Oaxaca, durante la presente década, se abrieron al público dos acervos que nos permiten profundizar en la vida escolar y la trascendencia de las acciones llevadas a cabo por la comunidad del IACAEO durante el siglo XX: el Archivo Histórico de la Universidad Autónoma Benito Juárez de Oaxaca (AHUABJO) y el Fondo Luis Castañeda Guzmán (FLCG), resguardado por la Biblioteca Juan de Córdova del Centro Cultural San Pablo. Castañeda fue 
estudiante, profesor y director del IACAEO desde 1928, así como un coleccionista de documentos que han permitido entender mejor la historia del estado de Oaxaca. Entre esos documentos se encuentra lo que se considera el archivo del comité estudiantil que llevó a efecto la huelga de 1936. La documentación que se resguarda en estos archivos nos permite matizar visiones que han calificado al instituto oaxaqueño de la primera mitad del siglo Xx como una institución de reputada religiosidad (Smith, 2009: 112).

La huelga de 1936 en el IACAEO ni siquiera se menciona en la principal obra historiográfica del instituto y la universidad oaxaqueña (Martínez, 2012), y apenas se dice algo de aquélla en el libro conmemorativo del cincuentenario de la conversión del instituto en universidad (Ruiz et al., 2006). Este silencio (debido en parte a que las fuentes eran inaccesibles) no sólo ha impedido valorar la importancia de esos acontecimientos en el desarrollo histórico de la institución, sino sus particularidades en el contexto de la política del gobierno de Cárdenas para la educación superior.

En el IACAEO se desarrolló, durante la primera mitad del siglo XX, una cultura participativa que se transmitió entre generaciones. Las sesiones científico-literarias, los concursos de oratoria, los organismos de representación estudiantil y la posibilidad que tenían los alumnos más avanzados de dar clases a los alumnos de niveles inferiores fueron oportunidades que permitieron construir una comunidad participativa.

Aquí veremos que, entre los profesores de 1936, se encontraban ex alumnos que habían participado en 1914 en un movimiento estudiantil en la casa de estudios oaxaqueña, durante la campaña de José Vasconcelos por la gubernatura en 1924, y en un concurso nacional de oratoria. Estos profesores respaldaron el movimiento contra el gobierno local y permitieron que fuera encabezada por los estudiantes.

La huelga permitió que la comunidad del instituto avanzara en la consecución de mayor autonomía respecto del gobierno estatal y su recuerdo hizo que sectores de la sociedad oaxaqueña que se manifestaron contra el gobierno estatal voltearan al colegio para que asumiera un papel protagónico en las protestas que dieron como consecuencia la caída de dos gobernadores en 1947 y 1952. En estos movimientos, un líder estudiantil de 1936, Luis Castañeda, tendría un papel relevante y la misma comunidad del IACAEO dirigiría a quienes pugnaban por la caída de Edmundo Sánchez Cano y Manuel Mayoral Heredia.

\section{Disciplina, oratoria y organización estudiantil en el IACAEO}

El 8 de enero de 1926, este instituto (aún no autónomo) cumplió 99 años. El periódico local El Mercurio del día siguiente dio cuenta del concierto que se realizó en los pasillos del edifico, pero, sobre todo, de la serie de tropelías que cometieron los alumnos durante este día de asueto: 
el conjunto de estudiantes distraía su ocio de que gozaron por la rememoración dedicándose a juegos diversos y maldades varias entre ellos. Antes de que el plantel se abriera, el grupo de jóvenes estacionados en la puerta, sediento de motivos de distracción, jugó entre ellos la designación del que habría de dirigir insinuaciones amorosas y requiebros a la primera dama que pasara [...]. Después, los jóvenes se dedicaron a la ingrata variedad de proporcionar baños forzados entre ellos mismos, tomando al elegido y sumergiéndolo por la fuerza en el estanque de agua. ${ }^{1}$

La indisciplina de los estudiantes continuaba ocho años después. En un documento dirigido a la Federación de Estudiantes Oaxaqueños (FEO), el secretario de la Sociedad CientíficoLiteraria solicitaba que el organismo reclamara al director justicia para cuatro estudiantes que habían sido expulsados del instituto por causa de una nota con "conceptos injuriosos" en el periódico estudiantil Eco. La petición argumentaba que la medida era contraria a la libertad de expresión protegida por el artículo 14 constitucional. Igualmente indicaba que, a lo sumo, los estudiantes ameritaban una amonestación porque el texto estaba escrito de forma contraria a la estética y el buen decir, pero no habían transgredido artículo alguno del Reglamento del IACAEO. Por último, mencionaba que, unos días antes, otro alumno había insultado a un catedrático cuando éste daba su clase y que no había sido sancionado. ${ }^{2}$

Si bien es posible encontrar en las fuentes casos de indisciplina, también lo es ver la otra cara de la moneda. La oratoria era una práctica muy apreciada por la comunidad. En aquel entonces, estudiantes del instituto lo representaban en los concursos anuales que organizaba el periódico de circulación nacional El Universal. En 1927, Raymundo Manzano Trovamala obtuvo el cuarto lugar en el concurso que premiaba al ganador con participar en un certamen internacional.

En 1929, Roberto Ortiz Gris obtuvo el primer lugar en el mismo concurso, superando al mexiquense y representante del Distrito Federal, Adolfo López Mateos ${ }^{3}$ (Tardiff, 1961: 201216). Entre 1931 y 1947, este concurso nacional se suspendió, por lo que los oradores oaxaqueños que sobresalían ya no pudieron medirse con sus pares del resto del país, aunque sus habilidades se siguieron desarrollando en diversos ámbitos de la vida escolar.

\footnotetext{
1 "El aniversario de la fundación del Instituto", El Mercurio, 9 de enero de 1926, p. 3.

2 AHUABı, Guillermo Martínez León/Federación de Estudiantes Oaxaqueños, 9 de mayo de 1935, caja 100, Asociaciones estudiantiles, 1935.

${ }^{3}$ Adame (2014). En este artículo se indica que los biógrafos de López Mateos han dado por hecho el triunfo del mexiquense en el concurso de 1929, sin embargo, los periódicos indican que fue Roberto Ortiz Gris el ganador. Enrique Krauze, en su serie televisiva México Siglo xx, inicia un programa dedicado a López Mateos indicando que fue el primer presidente campeón de oratoria. Al ponderar su destreza en el arte del buen decir, indica que ganó múltiples concursos de esta disciplina. También indica que se inscribió al concurso de El Universal presentando un acta de nacimiento falsa. Respecto del resultado del concurso nacional señala: "Al parecer por una decisión arbitraria de los jueces se le dio el segundo lugar" y después sus compañeros lo pasearon en hombros. La crónica detallada del concurso se encuentra en el texto ya aludido de Tardiff (Krauze, 1998).
} 
Además de participar en los concursos nacionales de oratoria, los alumnos desempeñaban funciones clave en las sesiones científico-literarias que se celebraban en el instituto desde 1908. En el archivo histórico de la UABJo hay crónicas de sesiones realizadas de 1931 a 1935 escritas por estudiantes designados por el director o por el alumno secretario de la sociedad científico-literaria.

A través de estas fuentes, se sabe de las actividades realizadas por la comunidad a las ocho de la noche de cada sábado y en fechas especiales, como las fiestas patrias o en la conmemoración de la toma de La Bastilla. Estas sesiones constituían una oportunidad para desarrollar habilidades relacionadas con la oratoria, la escritura y las artes (Padilla, 2004: 61). La asistencia a las sesiones era obligatoria para todos los alumnos, aunque en 1935 el director Julio Bustillos Montiel estaba preocupado por el ausentismo y comenzó a implementar algunas medidas para disminuirlo.

Por lo regular, cada sesión era presidida por el director del instituto y, en casos extraordinarios, por el gobernante del estado en turno. Se invitaba a las principales familias de la sociedad oaxaqueña, al gobernador y a los miembros de su gabinete. En uno u otro caso se tocaba una campanilla para dar inicio y para señalar el paso entre un acto y otro. Las sesiones incluían al menos dos actos musicales que podían ser ejecutados por estudiantes, la banda del gobierno del estado o la big band de la policía. Asimismo, se leía alguna poesía y se presentaban disertaciones de profesores o estudiantes sobre un tema histórico, literario o social.

En ocasiones, había una sección de asuntos generales en la que se podían tratar temas relacionados con la marcha del IACAEO. También podía suceder que se invitara a una personalidad del ámbito artístico, literario, político o científico de visita en la ciudad, cuya presentación podía ser el único acto de la sesión. Esos fueron los casos, en 1933 y 1935, de las sesiones en las que participaron el cómico capitalino Pepe Peña y el escultor catalán nacionalizado estadounidense Urbici Soler. ${ }^{4}$

Las crónicas y otros documentos relacionados con asociaciones estudiantiles muestran la creciente participación de las mujeres en el instituto. Ellas realizaron algunas de las crónicas en 1931, ${ }^{5}$ año en el que fue creada una federación de alumnas ${ }^{6}$ que se dividió por la elección de la Señorita Oaxaca, previa a las fiestas del Cuarto Centenario de la Ciudad de

\footnotetext{
${ }^{4}$ AHUABjo, Juan Velasco Jr., "Una charla con Pepe Peña", Crónica", 5 de agosto de 1933. Sociedad Científico-Literaria, "Programa de la sesión del sábado 10 de agosto de 1935 organizado por el Centro Estudiantil 'Cultura' pro Universidad del Sur, en honor del eminente escultor catalán don Urbici Soler", 10 de agosto de 1935, Caja 100, Asociaciones estudiantiles, 1935.

${ }^{5}$ AHUABıo, Ofelia Garcés, "Crónica de Sesión Científico-Literaria del $1^{\circ}$ de agosto de 1931", Caja 100, Asociaciones estudiantiles, asociación científico-literaria, 1931.

${ }^{6}$ AHUABjo, "Designación de Heliodoro Díaz Quintas como Miembro Honorario de la Sociedad Femenina Estudiantil Donaji", 12 de marzo de 1932. Carmen Matos/Heliodoro Díaz Quintas, 26 de febrero de 1932, Caja 100, Alumnos, Asociaciones Estudiantiles, 1932.
} 
Oaxaca en 1932 (Traffano, 2012: 68-90). A partir de 1935, las mujeres presentaron trabajos en las sesiones científico-literarias ${ }^{7}$ y comenzaron a influir en la designación de cargos de importancia en el organismo estudiantil de la carrera de Comercio y Administración. ${ }^{8}$ En ese año, las primeras alumnas que se titularían como abogadas y como médicas a principios de los años cuarenta en el IACAEO ya se encontraban en la vocacional del mismo.

Los órganos de representación de los estudiantes eran otro ámbito de participación juvenil. El principal era el Congreso Local de Estudiantes de Oaxaca (CLEO). Surgido en 1921, este organismo representaba a los estudiantes ante las autoridades educativas, conseguía beneficios para los alumnos del instituto y, en algunas ocasiones, participaba en los conflictos políticos locales. En 1924, el CLEO manifestó su apoyo a la candidatura de José Vasconcelos a la gubernatura de Oaxaca, ${ }^{9}$ en medio de un proceso electoral repleto de irregularidades ( $\mathrm{Ra}-$ mírez, 2016). También era el enlace entre los estudiantes oaxaqueños y la CNE. Anualmente eran elegidos representantes de cada una de las licenciaturas y de la preparatoria. Los electos conformaban la mesa directiva que fungía durante un año y cuyos cargos eran rotatorios.

Los cambios de la mesa directiva podían implicar problemas entre el alumnado. Por ejemplo, en 1928, el estudiante del último año de Jurisprudencia, Raymundo Manzano Trovamala, fue impugnado como presidente del congreso y derrocado por dos de sus colegas, debido a que no organizó ninguna fiesta para recabar fondos ni generó publicaciones dirigidas al estudiantado. La destitución se realizó en una asamblea a la que asistió la mayoría de los 617 estudiantes de las carreras de Jurisprudencia, Medicina, Comercio, Telegrafía, Taquigrafía, Instrumentista y Obstetricia; así como la Preparatoria, y en la que fue evidente el control que tenían sobre la comunidad los once futuros abogados que se destacaban como hábiles oradores. ${ }^{10}$ Ese año, el presidente del CLEO logró la aprobación de un reglamento para organizar las actividades del organismo que dirigía. En 1930, la elección se llevó a cabo por medio de un sistema de partidos. El proceso terminó en conflicto, por lo que el director decidió que cada partido dirigiera al Congreso durante medio año, alternándose en periodos de tres meses. ${ }^{\text {.1 }}$

7 AHUABJO, Elia Olivera, "Las costumbres de la mujer moderna han roto con la moral tradicional", 30 de marzo de 1935, Caja 100, Asociaciones estudiantiles, 1935. Elia Olivera sería la primera médica titulada en el IACAEO en la primera mitad de la década de los años cuarenta.

${ }^{8}$ AHUABıo, Gustavo Pérez y Ana María Sumano, "Acta de renovación de la Mesa Directiva de la Sociedad de Alumnos de la Facultad de Comercio y Administración", 27 de marzo de 1935, Caja 100, Asociaciones estudiantiles, 1935.

${ }^{9}$ AHUABıo, "Sesión Extraordinaria del IV Congreso Local de Estudiantes", 5 de julio de 1924, Caja 100, Asociaciones estudiantiles, 1924.

${ }^{10}$ AHUABJO, "Acta de asamblea realizada en el Instituto para cambiar a la mesa directiva del CLEO", 16 de febrero de 1928, Caja 100, Asociaciones estudiantiles, 1928.

${ }^{11}$ AHUABıo, "Acta con motivo del convenio celebrado entre los líderes de los partidos 'Revolucionario' y 'Demócrata", 28 de febrero de 1930, Caja 100, Asociaciones estudiantiles, 1930. 
En 1934, el CLEO se convirtió en la Federación de Estudiantes Oaxaqueños (FEO), manteniéndose cercana a las directrices de la CNE y en el mismo año se creó la Federación de Estudiantes Socialistas de Oaxaca (FESO), que rivalizó con la primera por buscar la representatividad de los alumnos. El surgimiento de organizaciones estudiantiles socialistas en el país y las consecuentes rivalidades, como en el caso de Oaxaca, tienen como antecedente la polémica Caso-Lombardo en el marco del Primer Congreso de Universitarios de México, organizado por la CNE en 1933. Ahí se discutió si las universidades e instituciones de educación superior debían asumirse como socialistas o ser ajenas a cualquier ideología (Hernández, 1969: 87-89). Las consecuencias de esta polémica se notaron en el desarrollo de la CNE. Desde la segunda mitad de los años veinte, la confederación organizaba anualmente el Congreso Nacional de Estudiantes. El de 1927 se realizó en el IACAEO, que celebraba su primer centenario.

La Confederación fue parte de las jornadas de 1929, que desembocaron en la autonomía de la Universidad Nacional. A principios de los años treinta, Ciriaco Pacheco (un ex estudiante del instituto oaxaqueño que salió del mismo por una huelga en 1927 y continuó sus estudios en la Universidad Nacional) escribió apuntes que nos muestran cómo se organizaron los estudiantes en el seno de la CNE durante la tercera década del siglo ( $\mathrm{Pa}-$ checo, 1980).

Como resultado de lo sucedido en el congreso universitario de 1933, el control del organismo nacional estudiantil lo tomaron estudiantes que estaban en contra de la educación socialista, entre los que estaban integrantes de la Unión Nacional de Estudiantes Católicos (UNEC), quienes pugnaban por la autonomía de las instituciones de educación superior (Loaeza, 1999: 128). Entre 1934 y 1935, se creó la Confederación de Estudiantes Socialistas de México (CESM), cuyo principal objetivo era que las instituciones de educación superior asumieran el socialismo como ideología dominante, al tiempo que acusaba a la CNE de autonomista, católica y reaccionaria. A su vez, las universidades de Guadalajara y Michoacán se asumieron como socialistas (Gómez, 2003: 189-191).

El siguiente año, la comunidad del Instituto Científico y Literario del Estado de México, en un movimiento que integró a autonomistas y socialistas, llevó a cabo una huelga ante una serie de acciones autoritarias del gobierno estatal, dentro del proceso en el que se pugnaba por la autonomía (Civera, 1988: 36-39).

Un ejemplo de cómo se manifestó la polémica antes mencionada en el IACAEO se observa en la sesión científico-literaria del 13 de abril de 1935. El último punto del orden del día era asuntos generales. Luis Castañeda Guzmán, estudiante del último año de la Vocacional de Jurisprudencia, tomó la palabra para denunciar a dos miembros de la mesa directiva de la FESO por no haber pagado el premio de segundo lugar a un poeta poblano que había sido laureado en los juegos florales del año previo. Para defenderse, los aludidos acusaron a Castañeda de haber robado dinero durante su gestión como tesorero de la FEO un año antes. 
Los gritos, aplausos y abucheos indicaban que los asistentes daban más crédito a las palabras de Castañeda. Entonces, los estudiantes socialistas trataron de desacreditarlo diciendo que su padre era sacerdote católico. Un alumno que formaba parte del público le recordó a uno de los estudiantes socialistas que su hermano también era sacerdote. Otro le dijo a uno de los integrantes de la FESO que, cuando se cerraron los templos católicos, su familia asistía a misas en un domicilio privado. Inmediatamente, el director dio por concluida la sesión.12

\section{El subsidio y el inicio de la huelga}

Durante el porfiriato, el IACAEO recibía del gobierno del estado el más importante subsidio que se daba a una entidad educativa en la entidad. Durante la época en que se dio la intervención carrancista en Oaxaca (1916-1920), la casa de estudios cerró por un año y, al reabrir en 1917, dejó de recibir recursos. A partir de 1921, el subsidio fue limitado, por lo que en 1927 se implementaron cuotas de inscripción y colegiaturas mensuales a los alumnos inscritos, recursos que se destinó a la compra de materiales para las actividades académicas, deportivas y artísticas de la comunidad.

En julio de 1935, el gobierno estatal había dejado de cumplir con su obligación de suministrar el subsidio, de por sí escaso, que servía para pagar el salario de los maestros. A finales de ese año, la Asamblea de Profesores, órgano de dirección del instituto que se conformaba con su planta docente y era encabezada por el director, manifestó su preocupación por la situación económica. En diciembre se llegó al acuerdo de solicitar recursos al gobierno federal, a través de una instancia cuya creación se había anunciado recientemente: el Consejo Nacional de Educación Superior e Investigación Científica (CNESIC). ${ }^{13}$

Este organismo se creó a raíz de la tensión entre el gobierno federal y las universidades durante la gestión de Lázaro Cárdenas. Algunos sectores consideraban que las carreras liberales existentes en la Universidad Autónoma de México y en las instituciones de educación superior de los estados no eran acordes con la necesidad de formar trabajadores industriales, capacitados técnicamente, solidarios con sus semejantes y dispuestos a apostar por el progreso nacional. Sin embargo, la Secretaría de Educación Pública (SEP) no tenía forma de tomar decisiones o intervenir en las instituciones universitarias. Cárdenas creó el CNESIC, el cual inició funciones en enero de 1936. Estaba conformado por catorce destacados académicos y profesionistas y buscaba, entre otras cosas, generar iniciativas que permitieran la

\footnotetext{
${ }^{12}$ AHUABJO, Leopoldo Gatica B., "Crónica de la sesión 'científico-literaria' verificada el 13 del presente", 27 de abril de 1935, Caja 100, Asociación Científico-Literaria, 1935.

${ }^{13}$ BIJC-FLCG, "Actas de Reunión y Circulares giradas por el director a los Profesores del Instituto", diciembre de 1935. Caja 38, 1933-1935.
} 
intervención del gobierno federal en los institutos y universidades del interior del país ( $\mathrm{Gu}$ tiérrez, 2009: 83-85), que hasta ese momento tenían como principal guía a la Universidad Autónoma de México.

El primer presidente del Consejo fue Enrique Díaz de León y su secretario Enrique Arreguín. Como parte de las acciones del nuevo organismo, se creó en 1936 el Instituto Politécnico Nacional, cuya fundación significó una ruptura con el modelo universitario originado desde mediados del siglo XIX (Quintanilla, 2008: 16). A diferencia de otras instituciones que vieron la creación del CNESIC como un riesgo a su autonomía (Riquelme, 2010: 25), el IACAEO la tomó como una posibilidad de solución a su falta de recursos, por lo que la Asamblea de profesores le envió una solicitud de subsidio.

El tema de los salarios no generó problemas mayores en el IACAEO durante la segunda mitad de 1935 y hasta julio de 1936, aunque parece haber contribuido al ausentismo de los profesores. La mayoría de los docentes tenía otras actividades, ya fuera en la administración pública o de orden privado. Otros eran alumnos del propio instituto, seleccionados porque mostraban dominio de determinadas materias. Se les condonaba el pago de sus cuotas y se les pagaba por impartir clases. Un padrón de profesores de 1936 nos muestra que dieciséis de los sesenta y un profesores eran alumnos; es decir, una cuarta parte de la planta docente, lo que nos habla de la importancia que tenía la participación estudiantil no sólo en la FEO y las sesiones científico-literarias, sino también en la Asamblea de Profesores.

A finales de julio y principios de agosto de 1936, culminó el proceso electoral que resultó en la elección de Constantino Chapital como gobernador del estado (Smith, 2009: 113). El 31 de julio, el gobernador saliente, Anastasio García Toledo, decidió sustituir al abogado Julio Bustillos Montiel, director del IACAEO desde 1934, por el médico Joaquín B. Unda. Tanto el gobernador García Toledo como Bustillos Montiel habían sido estudiantes del instituto durante el movimiento de 1914, que logró la aprobación por decreto de los alumnos en el año en que el gobierno del estado había suspendido las labores educativas por causa de la ocupación estadounidense en Veracruz (Ruiz, 1998: 112). También habían vivido la difícil época del Instituto Libre durante la ocupación carrancista en el estado, en la que la voluntad y organización de profesores y alumnos hizo funcionar a la casa de estudios sin contar con subsidio alguno.

En los años veinte, a diferencia de García Toledo, Bustillos se convirtió en profesor habitual del IACAEO y, aunque ocupó cargos como el de diputado federal a fines de los años veinte y principios de los treinta, había procurado permanecer en la planta docente ( Aguilar, 2014a: 6-7). Si bien en 1935 como director había tenido problemas al censurar una publicación estudiantil, durante el desarrollo de su administración se ganó el respaldo de los estudiantes. La falta de suministro del subsidio (que ya cumplía un año) y la destitución del director hicieron que el 3 de agosto de 1936 los estudiantes se declararan en huelga. 
Ese día, se conformó el Comité para llevar a cabo la suspensión de labores, cuyo presidente fue José Manuel Santibáñez. También se nombraron dos representantes estudiantiles ante el gobierno estatal, uno de los cuales fue Luis Castañeda Guzmán. ${ }^{14}$ Por la noche, el Comité envió telegramas a la Ciudad de México para informar al presidente de la República, a la SEP y a la CNE el inicio de la huelga y los motivos de ésta, así como solicitar su intervención para que la solución fuera a favor de la causa estudiantil. Dos días después, el presidente del Comité giró un telegrama a Enrique Díaz de León, presidente del CNESIC, informando que los profesores del IACAEO habían celebrado una asamblea en la que confirmaban su apoyo a la huelga y solicitando que el organismo revisara los antecedentes que obraban en su poder (quizá refiriéndose a la solicitud de subsidio que se había acordado enviar en diciembre). También se le pedía su intervención para la pronta solución del conflicto. Estos términos fueron comunicados también a Gonzalo Vázquez Vela, secretario de Educación Pública.

José Manuel Santibáñez era estudiante del último año de Jurisprudencia y profesor de la secundaria nocturna. Al menos en dos sesiones científico-literarias se había manifestado cercano al marxismo. En una de ellas leyó un trabajo titulado "Por qué el socialismo no ha sido generalmente aceptado"15 y en otra "El anarquismo de Proudhon y el socialismo de Marx" ${ }^{16}$ La cercanía de Santibáñez con las ideas de izquierda y la gravedad de la problemática institucional facilitaron la unidad entre los estudiantes de la FEso y la FEO para llevar a cabo la huelga. Alumnos que participaban en organizaciones socialistas defendieron al movimiento en instancias externas. Un estudiante miembro de la FESO dirigió un telegrama a la CESM para denunciar que el Comité estatal actuaba de forma reaccionaria y contra la causa estudiantil. Otro estudiante que pertenecía al Comité Estatal de las Juventudes Socialistas de México envió un telegrama el 7 de agosto al secretario de Organización y Propaganda de la oficina nacional de dicho organismo, para informar que no había distinción ideológica en la huelga que había estallado el día 3.17

Un ejemplo de la importancia del desarrollo de habilidades para la oratoria en las sesiones científico-literarias, la participación en los organismos de representación estudiantil y la posibilidad de que los estudiantes tuvieran injerencia en la Asamblea de profesores fue el caso de Luis Castañeda Guzmán, representante de los alumnos ante las autoridades estatales. Castañeda ingresó a la preparatoria del instituto en 1928 y en 1936 cursaba el primer año

\footnotetext{
${ }^{14}$ BIJC-FLCC, "Nombramiento de los representantes alumnos", 5 de agosto de 1936, Caja 38, Institutos educativos, Huelga, Correspondencia, 1936

${ }^{15}$ AHUABıo, María Concepción Garcés, "Crónica de la sesión científico-literaria" 3 de febrero de 1935, Caja 100, Asociaciones estudiantiles, 1935.

${ }^{16}$ AHUABjo, Sociedad Científico-Literaria, "Programa de la sesión inicial del segundo semestre de 1935", 6 de julio de 1935, Caja 100, Asociaciones estudiantiles, 1935.

${ }^{17}$ BIJC-FLCC, "Telegramas de estudiantes en huelga a diversos destinatarios", agosto de 1936, Caja 38, Institutos Educativos, Correspondencia, Huelga, impresos, proyectos, 1936.
} 
de Jurisprudencia. Su formación inicial cercana a las parroquias donde ejercía el sacerdocio su padre y las sesiones científico-literarias en el instituto le ayudaron a desarrollar importantes cualidades como orador (Velasco, 1976: 109-114). Además, había sido miembro de las mesas directivas del CLEO y la FEO de 1930, 1934 y 1935, lo que le daba experiencia en la gestión y trato con las autoridades del instituto y sus compañeros. Desde 1934 era profesor de Historia y Literatura en la secundaria del Instituto, por lo que tenía voz y voto en la Asamblea de profesores. ${ }^{18}$

\section{La prensa oaxaqueña y la huelga}

La prensa local desempeñó un papel destacado en el conflicto. Por un lado, El Oaxaqueño difundía la visión del gobierno estatal sobre la huelga; por el otro, Oaxaca Nuevo procuró mantener cierta neutralidad frente al conflicto. A su vez, la revista Oaxaca en México, que comenzó a editarse en la capital mexicana en agosto de 1936 y estaba dirigida a los oaxaqueños ahí avecindados, tenía como principal preocupación que se salvaguardara la independencia que tenía el IACAEO respecto de la reforma educativa socialista:

Dos caminos vemos para la reanudación de la vida normal del Colegio Oaxaqueño: el primero marca la aceptación de un subsidio de la Federación, que ésta ha ofrecido gustosa, ayuda que cancelaría la dificultad económica, base del conflicto. La segunda solución descansa en un entendimiento rápido entre la autoridad local y los huelguistas, mediante una gran dosis de buena voluntad de parte de ambos lados [...]. Con la primera solución, la Federación, que tiende abiertamente a centralizar la enseñanza, haría del Instituto de Oaxaca una dependencia atada a las orientaciones, sistemas y modalidades de cartabón y la vida propia, libre, especial del colegio de Oaxaca moriría con todas sus tradiciones y laures conquistados. ${ }^{19}$

El Oaxaqueño acusó a la huelga de reaccionaria. Vista desde sus páginas, era una manifestación autonomista que buscaba atacar al régimen de la Revolución mexicana:

A semejanza de los sucesos de hace cerca de medio siglo en que México se dividió en dos bandos ideológicos, hoy estamos presenciando la lucha de dos ideologías en su afán decidido de conquistar México [...]. Es necesario que el Estado, con la cooperación de la Academia de profesores, de ideología no clerical ni retardataria oriente a ese Plantel para convertirlo en una garantía de forjador de profesionistas que respondan a las necesidades del servicio social [...]. Por esto nunca el Estado, representante

\footnotetext{
${ }^{18}$ AHUABJO, "Nómina de catedráticos del Instituto", 8 de mayo de 1936, caja 77, Dirección, Informes, 1931.

19 "Cómo pensamos", en Oaxaca en México, t. 1, núm. 2, México, 29 de agosto de 1936, p. 3.
} 
de las Instituciones, cumpliría con su deber, dejando, que a pretexto de una mal entendida autonomía, se orientara a los futuros profesionistas dentro de ideas dogmáticas, no científicas y dentro de normas egoístas de utilitarismo y provecho personal. ${ }^{20}$

Esta forma de ver a los huelguistas era muy parecida a la que, en 1933, juzgaba a quienes se rebelaron contra las autoridades de la Universidad de Guadalajara. Quienes pugnaban por la implementación de la educación socialista aplicada por el rector Enrique Díaz de León (quien, como ya se indicó, fungía en 1936 como presidente del CNESIC) acusaban a la Iglesia de estar detrás del conflicto y tildaban de reaccionarios a sus contrarios (Dorantes, 1993: 185).

El Oaxaqueño descalificaba la huelga por cualquier motivo, como los actos de indisciplina de los estudiantes. ${ }^{21}$ Igualmente, promovía algunas ideas semejantes a las de quienes estaban a favor del cierre de la Universidad Autónoma de México por considerar que las carreras liberales ya no eran útiles a la sociedad y era necesario, para el caso de Oaxaca, abrir lo que denominaba la Universidad del Sureste, con carreras acordes con las necesidades del momento.22 Este periódico era dirigido por Roberto Ortiz Gris, abogado egresado del instituto y a quien los estudiantes en paro acusaron de haber vendido la huelga de 1927 contra la implementación de cuotas, que tuvo como uno de los resultados la salida del instituto de al menos dos estudiantes que continuaron sus estudios en la capital del país.

Ante las acusaciones del gobierno del estado y El Oaxaqueño respecto de que la huelga era contraria a los principios de la Revolución, los miembros del Comité invirtieron buena parte de sus esfuerzos en explicar sus motivos y congraciarse con el gobierno federal. Mantuvieron comunicación mediante cartas y telegramas con el presidente de la república, el CNESIC, los estudiantes oaxaqueños que vivían en la Ciudad de México y la CNE para explicarles los motivos de su causa. Además, a mediados de agosto, formaron la Central Única de Estudiantes Revolucionarios del Sur (CUERS), agrupación dirigida por los miembros del Comité de huelga, algunos miembros de la FEso y Esperanza Dea Velasco, alumna que destacaba por su participación en el organismo estudiantil femenino y en las sesiones científico-literarias. La CUERS se comprometía a fomentar en el instituto una orientación obrero-campesina, bajo los principios del materialismo histórico.. ${ }^{23}$ Además, Santibáñez procuraba mantener informado a Enrique Arreguín, secretario del CNESIC sobre la situación.

Dentro del instituto, la huelga también tuvo detractores. Por ejemplo, Jorge Fernando Iturribarría, que estudió la preparatoria e inició sus estudios de abogado en el instituto, pero

20 "Crónicas y comentarios. Función social del Instituto", El Oaxaqueño, Oaxaca, 18 de agosto de 1936, p. 3.

21 "La Dirección General de Educación en el conflicto estudiantil", El Oaxaqueño, Oaxaca, 12 de agosto de 1936, p. 1.

22 "Las organizaciones obreras del Estado piden la Universidad Socialista", El Oaxaqueño, Oaxaca, 20 de agosto de 1936, p. 3 .

${ }^{23}$ BIJC-FLCC, "Manifiesto de los estudiantes del Instituto al pueblo de México", 16 de agosto de 1936, Caja 38, Institutos Educativos, Correspondencia, Huelga, impresos, proyectos, 1936. 
no concluyó por dedicar más tiempo a las lides periodísticas y por ostentar cargos en diversos periodos gubernamentales en el estado ( Aguilar, 2014b: 5). Al momento de la huelga, Iturribarría se desempeñaba en la casa de estudios como profesor de historia en la secundaria y en la vocacional, así como secretario. Rafael Márquez Toro, secretario del Despacho del Gobierno del Estado, también era profesor en la carrera de Jurisprudencia y tuvo que lidiar con el problema tanto en palacio de gobierno como en las asambleas de la Academia.. ${ }^{24} \mathrm{El}$ profesor José Guillermo Toro, poco tiempo antes de la huelga, había dejado sus cátedras relacionadas con la lengua española en manos de su hijo Guillermo Toro Jr., quien también fue un detractor de la huelga..$^{25}$

\section{Las peticiones y la negociación}

El primer pliego petitorio de los huelguistas solicitaba que el artículo 16 de la Ley Orgánica, que daba poderes plenos al Ejecutivo del estado respecto de su reglamentación, fuera derogado; que los sueldos de los catedráticos y empleados fueran cubiertos desde la fecha en que habían dejado de pagarse; que el director saliente rindiera cuentas; que se concediera la autonomía plena al plantel a partir de una propuesta de los estudiantes; que el director fuera designado con el acuerdo de la Academia de profesores y los estudiantes y que se continuaran las gestiones con el gobierno federal para obtener un subsidio. ${ }^{26}$

La petición de la rendición de cuentas del director saliente no volvió a aparecer en el proceso de huelga, por lo que considero que se incluyó con el fin de desvincular a los huelguistas del aludido Bustillos Montiel. Como se verá más adelante, Bustillos fue partícipe de las conmemoraciones de la huelga que se realizaron al concluir y, dos años después, por lo que no se percibe que existiese la intención de los alumnos por auditarlo. El membrete del documento con las peticiones decía "Consejo Universitario de Profesores y Alumnos del Instituto de Ciencias y Artes del Estado", lo cual revela que la mayoría de los profesores y alumnos estaban de acuerdo con la huelga. Durante ésta, los docentes que no eran alumnos

\footnotetext{
${ }^{24}$ BIJC-FLCC, Comité de Huelga Estudiantil del Instituto/Juan Aranda, Esteban Silva y Escobar y Jorge Fernando Iturribarría", 5 de septiembre de 1936, Caja 38, Institutos Educativos, Correspondencia, Huelga, impresos, proyectos, 1936. Aranda y Silva eran alumnos que también fungían como profesores en la carrera de Contaduría y Administración y en la Vocacional.

${ }^{25}$ BIJC-FLCC, José Guillermo Toro/Manuel Matos director del Instituto, 7 y 11 de septiembre de 1936, Institutos Educativos, Correspondencia, Huelga, impresos, proyectos, 1936.

${ }^{26}$ BIJC-FLCG, "Al gobernador del estado, se le indican las peticiones de los estudiantes en huelga", 3 de agosto de 1936, Caja 38, Institutos Educativos, huelga, correspondencia, impresos, proyectos, 1936.
} 
se mantuvieron aparentemente al margen o, como diría al respecto Raymundo Manzano Trovamala en su informe como director en 1940, fueron "atingentes y discretos". 27

Las peticiones del Comité de huelga tocaban, ya fuera directa o indirectamente, la cuestión de la autonomía. Si bien la Ley Orgánica de 1931 la reconocía, existían candados para que el gobierno del estado mantuviera su injerencia en el IACAEO: el director era designado por el gobernador con la ratificación del Congreso, su presupuesto era aprobado entre el Ejecutivo y el Legislativo estatal (Sánchez y Ruiz, 2014: 109-112) y los planes de estudio, el calendario escolar y el de exámenes debían ser aprobados por la Cámara de Diputados.

Los estudiantes modificaron sus pretensiones en el transcurso del movimiento. El 13 de agosto, el Comité de huelga envió al gobernador una propuesta de nueva Ley Orgánica que aglutinaba los deseos de autonomía mostrados en el pliego petitorio original, arropados con un discurso en el que se planteaba la búsqueda del beneficio, tanto para el instituto como para el proletariado. La propuesta indicaba en el artículo 11 que el director sería nombrado por el Consejo Técnico, el cual estaría conformado por profesores y alumnos electos por sus pares, por lo que prácticamente el gobernador quedaría sin injerencia. El 16 de agosto, ya constituidos como CUERS, los huelguistas plantearon nuevas formas de organización de las carreras que se impartían en el instituto, al cual ya se referían como universidad.

Al principio, las peticiones se concentraron en la autonomía y en el pago del subsidio atrasado por el gobierno estatal. A la mitad de la huelga, se le dio más peso a la autonomía, con matices de discurso socialista, y hacia el final los estudiantes se concentraron en el pago del adeudo y en la defensa de cuatro profesores señalados por el gobierno como reaccionarios. Estas modificaciones se debieron en parte a la intervención del CNESIC. Su presidente, Díaz de León, había promovido en 1933 la educación socialista durante su gestión como rector de la Universidad de Guadalajara. A su vez, Arreguín había sido rector de la Universidad Michoacana en 1935.

En un intercambio de telegramas entre el 24 y el 25 de agosto, Arreguín informaba a Santibáñez que el gobernador había aceptado la totalidad de proposiciones y prometía que, al día siguiente, enviaría soluciones concretas. El día 25, Santibáñez se mostró inconforme con la propuesta del gobernador y le respondió a Arreguín que no había razón alguna para que Joaquín B. Unda, quien sustituyó a Bustillos, siguiera a la cabeza del instituto; la cantidad ofrecida por el gobernador no era la acordada previamente y le suplicaba que sostuviera las peticiones de los estudiantes. Un día después, Santibáñez le indicaba en otro telegrama a Arreguín que no podían permitir la separación de cuatro profesores y que el gobierno del estado regresaba a una propuesta de pago hecha los primeros días de la huelga. También le

\footnotetext{
${ }^{27}$ AHUABJO, "Informe de actividades del director Raymundo Manzano Trovamala al gobernador Constantino Chapital", 5 de octubre de 1940, Caja 77, Dirección, Informes 1940.
} 
informaba que una comitiva de trescientos estudiantes saldría a la ciudad de México para hablar con el presidente Cárdenas. ${ }^{28}$

Durante la huelga, los estudiantes tomaron el edificio, organizaron grupos de vigilancia, consiguieron descuento en el tren a la Ciudad de México, con el fin de enviar una comitiva que hablara con Cárdenas en torno al conflicto y entraron en comunicación con diversos sectores de la sociedad oaxaqueña que los apoyaron en un mitin realizado el 30 de agosto.

\section{La solución del conflicto}

A finales de agosto, las cartas y telegramas del Comité de Huelga a Arreguín mostraban cada vez mayor desesperación por parte de los estudiantes. Se sabía que pronto se resolvería el conflicto con el reconocimiento de la deuda por parte del gobierno del estado y la reinstalación de los profesores cuya destitución, según los estudiantes, era pedida por Rafael Márquez Toro, profesor del IACAEO y secretario del despacho del gobierno estatal.

El 2 de septiembre, se reunió la Academia de profesores convocada por su decano. Asistieron 47 profesores y el primer punto resuelto representó un logro para las peticiones autonomistas de los estudiantes. Después de que el gobernador del estado era quien nombraba al director del instituto sin mediar participación de su comunidad, en esta ocasión los profesores y alumnos eligieron una Comisión Permanente que se encargaría de dirigir al instituto al terminar la huelga. Dicha Comisión estaría conformada por cinco profesores y tres estudiantes. El gobernador elegiría de entre los primeros al nuevo director. En la votación fueron electos los médicos Manuel Matos, Manuel Canseco Landero, Fernando Bustillos y los abogados Alfredo Castillo y Manuel Aguilar y Salazar.

En el siguiente punto del orden del día, Rafael Márquez Toro informó que el gobierno del estado ya había puesto a disposición de la Academia la cantidad de \$5,000 como anticipo del pago total de la deuda y entregado al decano una nota para realizar el cobro. El documento fue entregado a Canseco Landero para que la Comisión cobrara en la Dirección de Rentas. Después, Luis Castañeda Guzmán, profesor de secundaria y representante estudiantil ante el gobierno estatal, solicitó a Márquez Toro que, como catedrático del instituto y en ese momento encargado del Poder Ejecutivo del Estado, informara a la Academia cómo haría el gobierno del estado para pagar al instituto los \$25,000 que restaban del adeudo que el gobierno tenía. Márquez Toro trató de convencer a sus colegas que la deuda no ascendía a la cantidad que Castañeda decía, sino que en las pláticas con el CNESIC se había acordado que era de aproximadamente $\$ 17,500$.00 y se pagaría en mensualidades de

\footnotetext{
${ }^{28}$ BIJC-FLCG, José Manuel Santibáñez/Enrique Arreguín, 26 de agosto de 1936, caja 38, Institutos Educativos, Correspondencia, Huelga, impresos, proyectos, 1936.
} 
$\$ 2,000$ o $\$ 3,000,{ }^{29}$ lo cual ponía en duda que se pagara la totalidad del adeudo, pues el periodo de gobierno de García Toledo concluiría en noviembre.

Los profesores elegidos para la Comisión Permanente eran cercanos a los estudiantes, afines a la causa de la huelga, egresados de la misma casa de estudios y algunos habían sido partícipes de la cultura participativa del IACAEO. Manuel Matos era uno de los profesores más antiguos. Aguilar y Salazar, cuñado de Luis Castañeda, había egresado del instituto en la época de la ocupación carrancista, y en 1921 fue electo diputado local para la legislatura que redactó la Constitución estatal aún vigente.

Canseco Landero había sido líder estudiantil en el instituto a fines de los años veinte. Bustillos era hermano del director depuesto a fines de julio y había sido parte de la mesa directiva del CLEO que apoyó a Vasconcelos en su campaña por la gubernatura del estado en 1924. Fernando Castillo era el padre de uno de los integrantes del Comité de huelga. El gobernador García Toledo nombró a Matos como director, cuya gestión duraría hasta mediados de 1937, cuando la nueva Ley Orgánica definió que el director debía ser nombrado por la asamblea de profesores a partir de una terna enviada por el gobernador del estado.

Los estudiantes eligieron a tres líderes del movimiento de huelga como sus representantes en la Comisión Permanente que dirigiría al IACAEO: Santibáñez, Castañeda y Rodolfo Sandoval. El primero egresó de aquél a finales de 1936, por lo que en enero del siguiente año, Castañeda, sin dejar de firmar como segundo miembro, lo relevó.

El 3 de septiembre, Santibáñez escribió a Díaz de León y a Arreguín para informar sobre los avances y recordarles que la comunidad del instituto estaba en espera de las instrucciones que diera el Consejo para la reorganización del IACAEO. ${ }^{30}$ Aun cuando la casa de estudios oaxaqueña abrió la puerta para que el CNESIC interviniera en aquélla, no hay evidencia de que esto haya sucedido.

Entre los documentos de la representación estudiantil encontrados en el Fondo Castañeda Guzmán está un acta de conclusión de huelga elaborada por los integrantes del Comité que la dirigió. Un fragmento de ésta da visos de las formas de convivencia de los estudiantes y su tono a la vez juvenil y consciente de los alcances del movimiento que recién habían encabezado:

La gloriosa bandera, a cuyo reverso se encuentra adherida la presente constancia, y que [... tiene la palabra "AUTONOMÍA", ondeó en el asta del Instituto de Ciencias y Artes del Estado [...] como símbolo de la Huelga que los estudiantes del mismo Colegio decretaron [...] exigiendo del Cobierno de Tacha la Burra el cumplimiento de los

\footnotetext{
${ }^{29}$ BIJC-FLCC, "Acta de Asamblea de profesores del iacaeo", 2 de septiembre de 1936, Caja 38, Institutos Educativos, Correspondencia, Huelga, impresos, proyectos, 1936.

${ }^{30}$ BIJC-FLCG, Comité de Huelga Estudiantil/Enrique Díaz de León y Enrique Arreguín, 3 de septiembre de 1936, Caja 38, Institutos Educativos, Correspondencia, Huelga, impresos, proyectos, 1936.
} 
deberes que como tal tiene para con el Instituto. Esta bandera será símbolo de una juventud que no se vendió y que supo siempre estar a la altura de su deber [...]. La directiva del Comité de Huelga se formó de la siguiente manera: presidente Chepo Santibáñez (Chepo la Burra), Primer Secretario Alberto Velázquez (Tragaldabas Capeto Selassie), Segundo secretario el más cabezón del Instituto (Fernando Castillo) y Tesorero el "Mismo yo" Pierre Arnaud. La bandera queda en poder de Fernando Castillo como botín de la lucha que sostuvo en la azotea del Instituto en contra de la "Madre Conchita Santaella" y del enano Alfredo 'Pirrín' Castillo a quienes ayudaba en espíritu el Lucas Güicho Castañuelas y el gigante Goliat. Firman para constancia todos los que pueden hacerlo, y no lo hacen los demás, porque son muy brutos. ${ }^{31}$

La Comisión Permanente tuvo como tarea inicial reorganizar el calendario escolar, por lo que solicitó a la Cámara de Diputados que aprobara un aplazamiento de las fechas de exámenes finales. ${ }^{32}$ Después de una negativa, el Poder Legislativo aprobó el aplazamiento, probablemente por la intervención del gobernador electo, el coronel Constantino Chapital, con quien la comunidad del instituto tenía muy buena relación.

Otro problema por tratar era el relajamiento de la disciplina que se percibía desde antes de la huelga. Los profesores habían dejado de asistir a sus cátedras y los alumnos no respetaban el reglamento de la institución. La Comisión hizo un llamado a los estudiantes para cumplir con sus deberes y que no hostigaran a los detractores del movimiento estudiantil.33

En noviembre, el gobernador saliente, García Toledo, dejó de cumplir con los pagos acordados, lo cual fue informado por Santibáñez al CNESIC. ${ }^{34}$ Esta situación hizo que Chapital, el gobernante electo, se comprometiera a aumentar el subsidio estatal al IACAEO, lo cual zanjó la amenaza de que la huelga fuera reactivada. El aumento del subsidio estatal ${ }^{35}$ y el inicio del suministro federal en 1937 permitieron un pequeño aumento en el salario de los profesores y la continuación de las obras de restauración del edificio del instituto, dañado por los temblores de 1928 y 1931 (Calderón, 2004: 47-50), que habían quedado inconclusas desde $1935 .^{36}$

${ }^{31}$ BIJC-FLCG, Comité de huelga, "Acta de cierre de huelga", 5 de septiembre de 1936, Caja 38, Institutos Educativos, Correspondencia, Huelga, impresos, proyectos, 1936.

32 AHUABjo, Comité de Alumnos de la Comisión Permanente, "Solicitud de aplazamiento de exámenes", 11 de septiembre de 1936, Caja 100, Asociaciones Estudiantiles, 1936

${ }^{33}$ AHUABıo, Comité de Alumnos de la Comisión Permanente del Instituto, "Llamado del Comité de alumnos a sus compañeros a guardar disciplina", 8 de septiembre de 1936, Caja 100, Asociaciones Estudiantiles, 1936.

${ }^{34}$ AHUABjo, Comité de Alumnos de la Comisión Permanente del Instituto/Enrique Arreguín, 12 de noviembre de 1936, Caja 100, Asociaciones Estudiantiles, 1936.

35 En 1937, se realizó el convenio de federalización de la educación básica por el que el gobierno federal cubrió el 58.59 por ciento de los salarios de los maestros, lo que alivió las finanzas estatales. Infiero que esto permitió que el gobierno del estado incrementara el subsidio al Instituto (Martínez, 2004: 38).

${ }^{36}$ AHUABıo, "Informe de Manuel Matos al Superior Gobierno del 15 de septiembre de 1936 al 15 de marzo de 1937", 15 de marzo de 1937, Caja 77, Dirección, Informes, 1935-46. 
Unos días después del levantamiento de la huelga, la Comisión Permanente que dirigía al instituto organizó una ceremonia presidida por el director Matos y el ex director Bustillo Montiel, en la que se premió a quienes realizaron acciones destacadas durante el conflicto. Dos años después, una sesión científico-literaria conmemoró el aniversario del inicio de la huelga. Luis Castañeda, para entonces estudiante del tercer año de Jurisprudencia, fue el orador principal.

\section{Reflexiones finales}

Durante la primera mitad del siglo XX, los estudiantes del IACAEO vivieron una formación participativa fundamentada en el desarrollo de habilidades para la oratoria, la organización de sesiones científico-literarias, así como la elección y funcionamiento de órganos de representación estudiantil, entre otras actividades. Lo anterior los hizo potenciales agentes de cambio, tal como se demostró durante la huelga de 1936.

Este movimiento sucedió en un contexto en el que el gobierno de Lázaro Cárdenas buscaba intervenir con mayor decisión en la educación superior del país. Dicho interés, fundamentado en la reforma educativa socialista, no fue posible impulsarlo desde la Universidad Autónoma de México, por lo que se creó el CNESIC, instancia a la que el IACAEO acudió para gestionar un subsidio federal que permitiera reducir las dificultades económicas que vivía. La irresponsabilidad del gobernador oaxaqueño Anastasio García Toledo, al no cubrir el subsidio al IACAEO durante un año, propició una huelga estudiantil en agosto de 1936 y la comunidad del instituto vio en el CNESIC una instancia que ayudaría a solucionar el conflicto. Ante la solicitud de apoyo, los miembros del Consejo actuaron como mediadores y gestores de recursos federales. La forma como se modificaron las demandas y el tono de los huelguistas pudo deberse a que, quienes encabezaban el CNESIC eran dos de los más importantes impulsores de la reforma socialista en la educación superior: Enrique Díaz de León en la Universidad de Guadalajara y Enrique Arreguín en la Universidad Michoacana.

Por otra parte, el temor que expresó un sector de los oaxaqueños avecindados en la Ciudad de México respecto de que el gobierno federal aprovecharía el conflicto para llevar adelante la imposición de su política educativa y sus afanes centralizadores, a cambio de participar en el financiamiento de la institución, terminó por ser desechado. Si bien la Comisión Permanente reconoció la labor realizada por el CNESIC en la solución del conflicto abriendo sus puertas para recibir indicaciones para la reestructuración de la casa de estudios, éstas nunca llegaron y el IACAEO procuró resolver los problemas que tenía, ahora con subsidio federal y con el apoyo de un nuevo gobierno estatal afín. 
La huelga de 1936 fue considerada como exitosa por la comunidad del instituto, aunque en su desarrollo sus artífices tuvieron que adecuarse a los discursos y condiciones que la época y los otros actores plantearon. Los huelguistas oaxaqueños lograron que el gobierno del estado empezara a solventar la deuda que tenía con el instituto. Respecto de las demandas autonomistas, profesores y alumnos llevaron adelante la elección de la Comisión Permanente y tuvieron que ceder la designación del director al gobernador, al tiempo que se tuvo que esperar un año más para discutir una nueva Ley Orgánica.

La huelga generó avances en la obtención de la autonomía y la comunidad de la institución se movilizó cuando ésta se vio en riesgo. La Ley Orgánica del siguiente año planteó que la elección del director la realizaría la Academia de Profesores, considerando una terna que el gobernador le enviaba. Este mecanismo se reafirmó en la Ley Orgánica de 1943.

Por otra parte, la figura del Consejo Técnico fue novedosa en la Ley Orgánica de 1937 y su función fue vigilar la buena marcha del instituto con una representatividad que incluía a profesores y alumnos. En diciembre de 1946, el gobernador Edmundo Sánchez Cano intentó imponer una Ley Orgánica en la que dicha instancia desaparecía. La comunidad del instituto con el director al frente, consideró que esto vulneraba su autonomía y se unió a las manifestaciones populares de repudio contra el gobernador por la imposición de un código fiscal que afectaba a amplios sectores de la sociedad oaxaqueña. Éstas derivaron en la destitución del gobernador en enero de 1947.

\section{Fuentes}

Archivos

AHUABjo Archivo Histórico de la Universidad Autónoma "Benito Juárez" de Oaxaca; Biblioteca "Francisco de Burgoa", Oaxaca.

BIJC Biblioteca de Investigaciones "Juan de Córdova", Oaxaca.

FLCG Fondo Luis Castañeda Guzmán.

Hemerografía

El Mercurio.

El Oaxaqueño.

Oaxaca Nuevo.

Oaxaca en México.

Bibliohemerografía

Calderón Martínez, Danivia (2004), Paraninfo de la Universidad. Memoria de su restauración, Gobierno del Estado de Oaxaca, Oaxaca.

Dorantes, Alma (1993), El conflicto universitario en Guadalajara, 1933-1937, Secretaría de Cultura, Gobierno de Jalisco/INAH, Guadalajara, Jalisco. 
Gómez Nashiki, Antonio (2003), "El movimiento estudiantil mexicano. Notas históricas de las organizaciones políticas, 1910-1971", Revista Mexicana de Investigación Educativa, vol. 8, núm. 17, enero-abril, pp. 187-220

Gutiérrez López, Miguel Ángel (2009), "El Consejo Nacional de la Educación Superior y la Investigación Científica y la política de educación superior del régimen cardenista, 1935-1940", Perfiles Educativos, vol. 31, núm. 126, pp. 80-98.

Hernández Luna, Juan (1969), "Polémica de Caso contra Lombardo sobre la Universidad", Historia Mexicana, vol. 19, núm. 1, julio, pp. 87-104.

Lempérière, Annick (1994), "La formación de las elites liberal es en el México del siglo xIX: Instituto de Ciencias y Artes del estado de Oaxaca", Secuencia, núm. 30, septiembre-diciembre, pp. 57-94.

Loaeza, Soledad (1999), El Partido Acción Nacional: la larga marcha, 1939-1994, Fondo de Cultura Económica, México.

Martínez Vázquez, Víctor Raúl (2012), Breve historia de la Universidad Autónoma Benito Juárez de Oaxaca, Universidad Autónoma "Benito Juárez" de Oaxaca, Oaxaca.

Martínez Vázquez, Víctor Raúl (2004), "La educación en Oaxaca después de la federalización de 1937", Acervos. Boletín de los archivos y bibliotecas de Oaxaca, vol. 7, p. 38.

Pacheco Calvo, Ciriaco (1980), La organización estudiantil en México, Universidad Autónoma de Sinaloa, Culiacán.

Padilla, Antonio (2004), Tiempos de revuelo: juventud y vida escolar (El Instituto Científico y Literario del Estado de México, 1910-1920), Universidad Autónoma del Estado de Morelos/Miguel Ángel Porrúa, México.

Quintanilla, Susana (2008), "La educación en México durante el periodo de Lázaro Cárdenas 1934-1940", DIE-Cinvestav, México (Documentos DIE, 62).

Ramírez, Daysi (2016), "De elecciones y chanchullos: la contienda electoral por la gubernatura de Oaxaca en 1924", Legajos. Boletín del AGN, núm. 9, 8a época, año 3, enero-abril, pp. 11-84.

Riquelme Alcantar, Gabriela María Luisa (2010), "El Consejo Nacional de la Educación Superior y la Investigación Científica: una política educativa para los trabajadores, 1935-1938”, DIE-Cinvestav, México, tesis de doctorado.

Ruiz Cervantes, Francisco José (1998), "El pase por decreto. 1914-1968", Humanidades. Revista del Instituto de Investigaciones en Humanidades, núm. 3, Oaxaca, UABJo (agosto-diciembre), pp. 108-113.

Ruiz Cervantes, Francisco José et al. (2006), Testimonios del Cincuentenario, UABJo/Fundación Harp Helú, Oaxaca.

Sánchez Silva, Carlos y Ruiz Cervantes, Francisco José (2014), La UABJo y sus leyes fundamentales. 1827 1988, UABJo/Carteles Editores, Oaxaca.

Smith, Benjamin T. (2009), Pistoleros and Popular Movement. The Politics of State Formation in Postrevolutionary Oaxaca, Universidad de Nebraska, Lincoln.

Traffano, Daniela (2012), "Ligera crónica de una lucha galante" en Daniela Traffano y Salvador Sigüenza (coords.), Oaxaca 1932, Municipio de Oaxaca de Juárez, Oaxaca, pp. 61-90.

Velasco Pérez, Carlos (1976), Oaxaca de mis recuerdos, Universidad Autónoma "Benito Juárez" de Oaxaca, Oaxaca.

Recursos electrónicos

Adame, Ángel Gilberto (2014), "Octavio Paz, joven orador", Letras Libres, 11 de septiembre, documento html disponble en: <http://www.letraslibres.com/mexico-espana/octavio-paz-joven-orador>; (consulta: 18/11/2017).

Aguilar Aguilar, Rogelio (comp.) (2014a), "Julio Bustillos Montiel”, Indelebles. Publicación mensual, núm. 2, Casa de la Cultura Oaxaqueña, Oaxaca, documento pdf disponible en: <http://www.casadelacul- 
tura.oaxaca.gob.mx/wp-content/uploads/2016/04/Indelebles02.pdf>; (fecha de consulta: $1 / 05 / 2018)$.

Aguilar Aguilar, Rogelio (comp.) (2014b), "Jorge Fernando Iturribarría Martínez", Indelebles. Publicación mensual, núm. 08, Casa de la Cultura Oaxaqueña, Oaxaca, documento pdf disponible en: <http:// www.casadelacultura.oaxaca.gob.mx/wp-content/uploads/2016/04/Indelebles08.pdf>; (fecha de consulta: 8/11/2017).

Civera, Alicia (1988), "Política educativa del gobierno del Estado de México (1920-1940" México, Facultad de Filosofía y Letras, UNAM, tesis de Licenciatura en Pedagogía, documento html disponible en: <http://132.248.9.195/pmig2017/0073319/Index.html>; (fecha de consulta: 6/04/2018).

Krauze, Enrique (1998), "López Mateos. Historia de un seductor", Clío, México, documento html disponible en: <https://www.youtube.com/watch?v=Mp0j9Nj0-BM\&t=1047s>; (fecha de consulta: 4/12/2017).

Tardiff, Guillermo (1961), El verbo de la juventud mexicana a través de los concursos de oratoria de El Universal, El Universal, México, documento pdf disponible en: <http://archivo.eluniversal.com.mx/graficos/graficosanimadosl2/EU-Libro-Oratoria/files/assets/downloads/publication.pdf); (fecha de consulta: 14/02/2018).

Alejandro Arturo Jiménez Martínez es profesor de tiempo completo del Instituto de Ciencias de la Educación de la Universidad Autónoma "Benito Juárez" de Oaxaca (UABJO). Es Maestro en Historia Moderna y Contemporánea y estudiante del Doctorado en Ciencias del Departamento de Investigaciones Educativas del Cinvestav. Sus líneas de investigación versan sobre la formación de los intelectuales y de las instituciones educativas en México, siglo xx y los actores, procesos e instituciones educativas de los siglos Xx y XXI en México. Es coautor, junto con Néstor Montes y Olga Montes, de "Las políticas públicas de la modernización de la educación superior en la UABJO: 1992-2012", en De la política y la educación en Oaxaca, siglos XIX-XXI. Resultados de investigación en el IIHUABJO, Instituto de Investigaciones en Humanidades-UABJo, Oaxaca, 2015.

Recibido: 22 de septiembre de 2018

Aceptado: 19 de diciembre de 2018 